\title{
Continuous electrocardiographic recording in Prinzmetal's variant angina pectoris $A$ report of four cases
}

\author{
M. Guazzi, C. Fiorentini, A. Polese, and F. Magrini \\ From the Instituto di Clinica Medica II and Istituto di Ricerche Cardiovascolari, \\ University of Milan, Milan, Italy
}

\begin{abstract}
Four patients with Prinzmetal's variant angina pectoris were subjected to continuous electrocardiographic recording. In three of them several episodes of ST segment elevation unaccompanied by pain were recorded. In one patient, identical electrocardiographic alterations were observed both in presence or in absence of pain, while in the others a good correlation was evident between pain and severity of the electrocardiographic abnormalities. In two patients transmural myocardial infarction complicated the course of the angina. In contrast to the classical findings, in these patients the attacks of chest pain did not cease after the infarction, but became more frequent and severe. The electrocardiographic alterations of the anginal episodes occurred in the same myocardial areas involved by the infarction, so that a reversible superposition of electrocardiographic signs of acute ischaemia on those of recent necrosis was observed.

Continuous electrocardiographic recording provided the best means of investigation of these patients with the variant form of angina pectoris.
\end{abstract}

A variant form of angina pectoris was desscribed by Prinzmetal et al. (1959, 1960). The major differences from classic angina of effort were indicated as follows. (a) Pain occurs at rest and is not brought on by exercise; (b) generally it is longer in duration and often more severe; (c) it often occurs at approximately the same time each day; (d) the electrocardiogram during a severe attack shows ST segment elevations in those leads that reflect the ischaemic area and, with the cessation of pain, returns to its appearance before the onset of the attack. Even though a painstaking history often can be the key to the clinical diagnosis, electrocardiographic confirmation is advisable. A single electrocardiogram recorded during pain in a patient with Prinzmetal's variant angina can be misleading both because an acute myocardial infarction could erroneously be diagnosed, and because several important features can remain unobserved.

In the four patients with this variant form of angina here reported there are some unusual features; the continuous electrocardiographic recording provided an effective diagnostic means.

Received I5 December 1969.

\section{Report of cases}

Case I The patient, a 64-year-old farmer, was in good health until 1962. At that time he had sudden praecordial pain at rest of a few minutes' duration. An electrocardiogram taken the following day was normal. During the succeeding 6 years he had similar episodes of chest pain approximately twice a month, which were alleviated within a few minutes by sublingual nitroglycerin. In spite of the severe pain at rest he was able to accomplish his heavy physical labour without any discomfort. During the last Io months the pain gradually became more frequent and severe, mainly during the night, without relation to wakefulness or sleep. He was admitted to another hospital because of severe chest pain. No electrocardiogram was obtained during pain and the diagnosis remained undefined. At the time of the present hospital admission physical examination was unremarkable. Routine laboratory work, blood urea nitrogen, fasting blood sugar, serum transaminase determinations, and chest $x$-ray were within normal limits. The electrocardiogram at rest was normal (Fig. IA). Two step exercise test was unremarkable (Fig. IB). He remained asymptomatic during the first five days of his hospital stay and then began again to suffer daily attacks of anginal pain. He was asked to keep charting for one week, the time, severity, and circumstances of the attacks. They were from 8 to 12 in the 24 hours, usually of a duration of less 

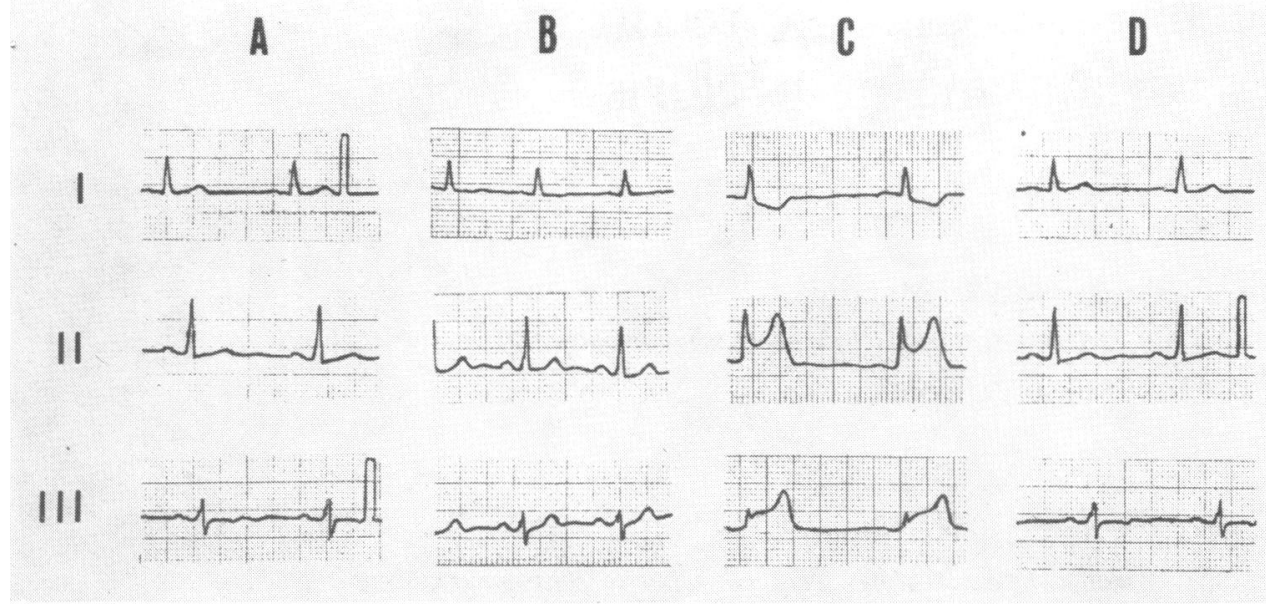

FI G. I (Case I) Leads I, II, and III taken when the patient was free of pain $(A)$, during the two-step exercise test $(B)$, during an episode of chest pain $(C)$, and 20 seconds after the pain had ceased. Note abnormal inverted $U$ in lead II $(D)$.

than one minute. He was especially likely to suffer pain at rest from 10:00 p.m. to 5:00 a.m. Repeated electrocardiograms were unremarkable. The pains were so brief that, in spite of many trials, an electrocardiogram could be taken only at the termination of an attack and was not altered from its usual appearance. The suspicion grew that the patient's symptoms were of psychoneurotic origin. At any rate, continuous electrocardiographic recording was decided. Recording of the three standard leads was done with the Hewlett-Packard 7868 A Recording System with simultaneous monitoring of lead I and lead II on the Hewlett-Packard 7803 A Monitor-Scope. The paper ran at a speed of $I \mathrm{~mm}$. per second during the periods free of pain and when no electrocardiographic abnormalities were evident on the Monitor-Scope. The speed was changed to 25 $\mathrm{mm}$. per second as soon as the patient had pain or electrocardiographic modifications were monitored. The patient held a push button for the remote control of a marker of the recording system. He was instructed to keep it pushed for the entire duration of the attack, so that a good time relation between pain and electrocardiographic alterations could be recorded. From ro:00 p.m. to 5:00 a.m. obvious ST segment elevations (Fig. IC) occurred on I6 separate occasions. Chest pain accompanied 7 out of these 16 episodes, whereas on 9 occasions the typical pattern of electrocardiographic changes was observed without the patient, though awake, experiencing any pain. There was a constant sequence of the electrocardiographic events: the $T$ wave first became taller in lead II and lead III, then ST segment elevation occurred and increased in extent. Each episode terminated with progressive $T$ wave reduction and return of the ST segment toward the isoelectric line. Twenty seconds after termination of an attack the electrocardiogram had reverted to its original appearance (Fig. ID). The electrocardiographic alterations not accompanied by pain usually were of brief duration (1-2 minutes). A correlation between severity of the attack and degree of the ST segment elevation was evident.

In this, as in the second patient here reported, a haemodynamic study was also performed (Guazzi et al., work in preparation). Arterial pressure, right atrial pressure, heart rate, and left ventricular ejection time were recorded; cardiac output was determined; total peripheral resistances, isovolumic contraction time, and mean rate of isovolumic pressure development were calculated. The circulatory changes during the periods of electrocardiographic abnormality with pain were, as shown by the measured parameters, qualitatively similar and quantitatively more obvious as compared to those occurring during the phases of ST segment elevation without pain.

The onset of pain was never before the electrocardiographic alterations. At the apex of some attacks rare extrasystoles were recorded.

Case 2 A 46-year-old workman, after a period of a few months during which he suffered short and rare episodes of substernal pain coming on at rest, awakened one morning with very severe praecordial pressure and pain associated with diaphoresis and faintness. He was admitted to another hospital where a diagnosis of acute anteroseptal myocardial infarction was made during the admission and documented by serial electrocardiographic changes. His course in hospital was uneventful. He became asymptomatic and returned to work. After less than one year he began to have episodes of aching substernal pain with radiation to the left wrist, occurring while at rest with a higher nocturnal frequency. He had been a heavy cigarette smoker, but even though he stopped smoking, the frequency of the attacks was not affected. At times he had to take as many as I5 


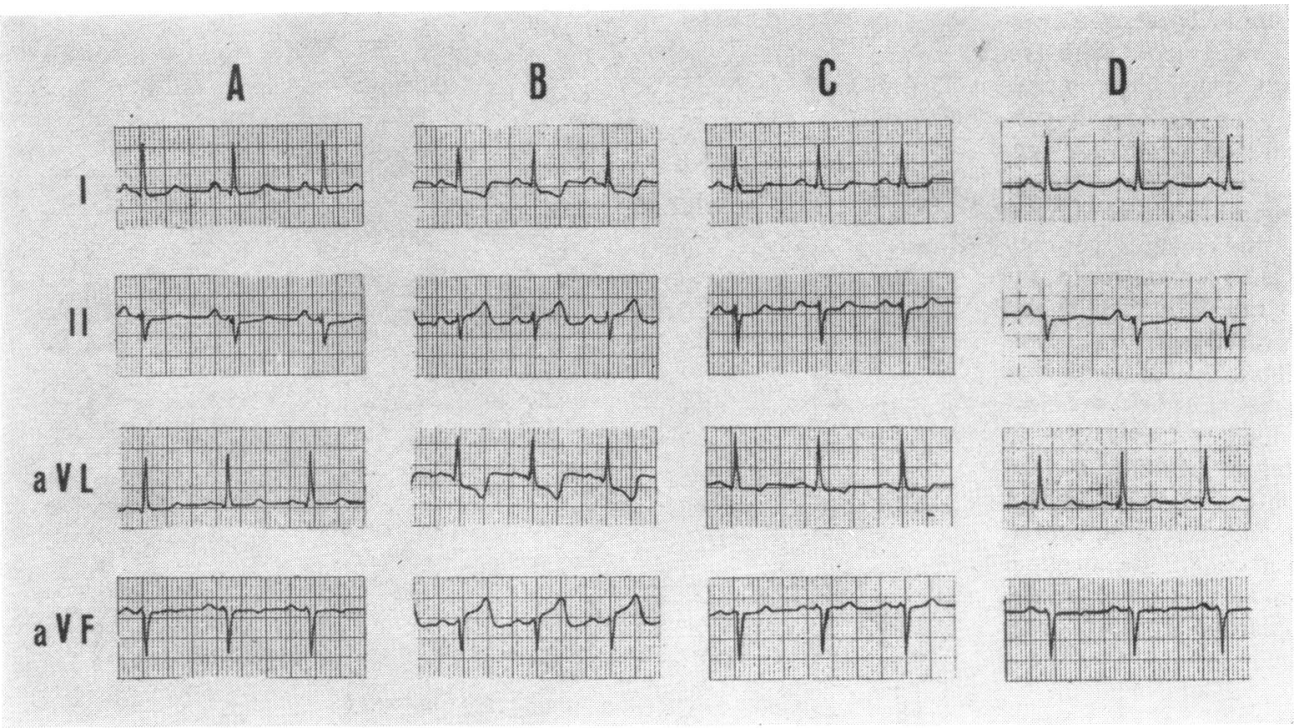

FIG. 2 (Case 2) Electrocardiogram taken in absence of pain $(A)$, during an attack of substernal pain $(B), 30$ seconds $(C)$, and 2 minutes $(D)$ after the end of the attack.

nitroglycerin tablets a day. Because of daily recurrences of these attacks, he came to the outpatient clinic. At the present hospital admission no significant abnormalities were noted on the physical examination. All laboratory studies were normal. An electrocardiogram showed an old antero-septal myocardial infarction and left ventricular hypertrophy. The patient was confined to bed and was given an anticoagulant drug. Charting of the attacks, taken for 7 days, showed a frequency of 5-6 episodes a day, mainly occurring during the night, without appreciable relation to meals, sleep, or wakefulness. The pain showed a definite waxing and waning period. The patient was then submitted to continuous electrocardiographic recording. Leads I, II, aVL, and aVF were recorded and monitored from $\mathrm{I}: \infty$ a.m. to 7:00 a.m., with the same procedure previously described. Fourteen episodes of electrocardiographic abnormalities were observed, each of them lasting only a few minutes, occasionally up to Io minutes. The electrocardiogram alterations were those reported in strip B of the Fig. 2 (obvious ST segment elevation in the inferior leads, reciprocal ST depression in the other leads, and no change in rhythm and atrioventricular conduction). Strip A of Fig. 2 reproduces the electrocardiogram recorded when the patient was free of pain, strips $C$ and $D$ are, respectively, taken 30 seconds and 2 minutes after the pain had stopped. Chest pain accompanied 7 of these 14 episodes. On 7 other occasions the typical electrocardiographic changes were recorded without the patient experiencing any disturbance, though he had been awake throughout the period of electrocardiographic recording. The ST segment elevation and the $T$ wave changes in absence of pain were similar in degree and duration as compared to those in the presence of pain. The onset of the pain in the was never before the onset of the electrocardiographic alterations. No changes in the cardiac rhythm or in the atrioventricular conduction were observed.

Circulatory modifications accompanied all of the episodes of ST segment elevation without significant qualitative and quantitative differences between the episodes with and those without pain.

Case 3 A 58-year-old man was admitted to hospital because of frequent attacks of chest pain at rest. He gave a history of rare episodes of retrosternal pain during the preceding ro months. Each episode had lasted less than 5 minutes and was not related to exertion. He consulted several physicians who could not find any evidence of cardiac abnormalities. When he was free of pain the electrocardiogram was normal. No electrocardiogram was obtained during the episodes of pain. He was not treated and continued at his occupation until 7 days before the present admission, when he had persistent ( 2 hours) and severe retrosternal pain, with radiation to the jaw and to the left upper extremity, from the shoulder to the fingers. Since that time the attacks of chest pain became much more frequent, occurring 5-6 times a day, while at rest, and lasting a few minutes.

On examination the blood pressure was $100 / 70$ $\mathrm{mm}$. Hg. The heart was not enlarged, the rhythm was regular, and the heart sounds were attenuated. Otherwise examination was unremarkable. Chest radiograph was within normal limits, serum aspartate aminotransferase was 53 units, the erythrocyte sedimentation rate (Westergren) was 2 I $\mathrm{mm}$. in I hour. An electrocardiogram showed $Q$ waves with $T$ wave inversion in leads II, III, and aVF consistent with a recent transmural diaphragmatic infarction (Fig. 3A), which probably occurred 
7 days before, when the patient had severe and persistent substernal pain. He was treated with rest in bed and an anticoagulant drug. Charting revealed a daily frequency of 8-12 episodes of substernal pain, lasting 30 seconds to 5 minutes, randomly distributed in the 24 hours. During 5 hours of continuous electrocardiographic recording 4 episodes of electrocardiographic changes were observed. In 3 of them, when the pain was mild, the ST segment was moderately raised in leads II and aVF, and the $T$ wave appeared spuriously improved in these leads (temporarily less negative). Whereas, at the height of a severe attack, the electrocardiogram showed obvious ST elevation in leads II and aVF and moderate elevation in lead III with spurious improvement of the $T$ waves (Fig. 3B); 30 seconds after the pain disappeared the electrocardiogram reverted to its original appearance (Fig. 3C). Even at the apex of the attack, no abnormalities in cardiac rhythm or in conduction were recorded.

Case 4 A 65-year-old mechanic was admitted to the hospital with a diagnosis of antero-septal myocardial infarction. His past history is significant because he had several separate episodes of chest pain, with radiation to the jaw and to the upper extremities, during the preceding three months. Each episode had lasted less than Io minutes, was relieved by nitroglycerin, and was not related to physical exertion; in fact, these episodes had occurred when he was relaxing after dinner. Six days before he was admitted to hospital, he awakened one morning with praecordial pressure and pain much more severe and persistent ( 3 hours) than usual. Since that time the pain had become more frequent, with 3-4 daily recurrences and he decided to come to the outpatient clinic. A diagnosis of recent anteroseptal myocardial infarction was made during the admission. Physical examination was unremarkable. Routine laboratory studies, fasting blood sugar, blood urea nitrogen, and chest $x$-ray were normal. Serum aspartate aminotransferase was 50 units. The erythrocyte sedimentation rate was high. The electrocardiogram on admission revealed $Q$ waves in leads $V_{I-2}$, ST segment elevation in leads $\mathrm{VI}_{\mathrm{I}}$ 4, and deep $\mathrm{T}$ wave inversion in leads $\mathrm{VI}-5$, consistent with antero-septal myocardial infarction (Fig. 4A). The patient was confined to bed and given heparin. During his hospital stay he was asymptomatic for the first 7 days and then attacks of substernal chest pain reappeared twice or three times a day, sometimes in the late afternoon, but more frequently in the early morning. Nitroglycerin given during the pain would usually terminate the attack. Five episodes of electrocardiographic abnormalities were recorded during five hours of continuous electrocardiographic recording. Only one of them was accompanied by aching substernal pain, whereas, on four occasions, electrocardiographic abnormalities were recorded without the patient suffering any pain, though constantly awake. The pattern of the development of the electrocardiographic changes was as follows: the negative $T$ waves first became upright in leads $\mathrm{VI}-5$, then $\mathrm{ST}$ segment

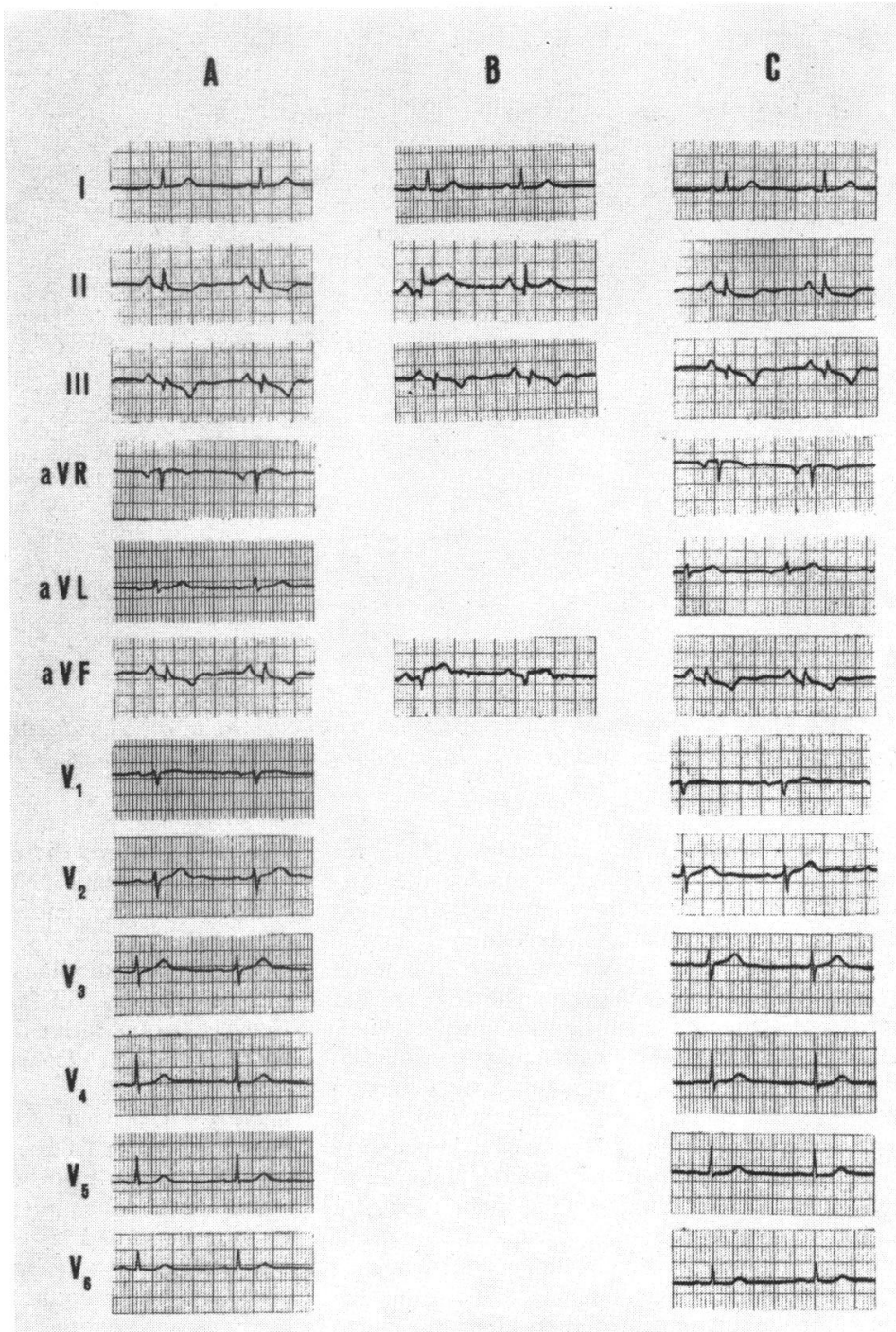

FIG. 3 (Case 3) Electrocardiogram of a patient with diaphragmatic myocardial infarction recorded in the absence of pain $(A)$, during severe spontaneous pain $(B)$, and 30 seconds after the pain had ceased $(C)$.

elevation occurred in leads V2-5 together with some increase in the amplitude of the $R$ wave in leads $V_{3}-5$. Fig. 4 reproduces the electrocardiogram of this patient recorded when he was free of pain (A), during the pain (B), and 50 seconds after the pain subsided (C). The ST segment and $T$ wave changes in the absence of pain were shorter in duration and a little less obvious, though still very clear, as compared to the changes recorded during the painful attacks. No cardiac rhythm or conduction abnormalities were observed. 


\section{Discussion}

In these patients there are many features which are described as typical (Prinzmetal et al., 1959, 1960) of the variant form of angina. The patients had pain at rest, not precipitated by exercise or emotion, with the classical distribution of angina pectoris. On all occasions the attacks were accompanied by striking ST segment elevations.

Pain in this variant form of angina has been described as more severe and longer than in classic angina (Prinzmetal et al., 1959); however in these patients it was, when occurring, brief in duration, seldom lasting more than 10 minutes.

Robinson (1965) reported a patient who sometimes showed ST segment elevation in the absence of chest pain. This feature was observed in 3 of the patients reported here, and in 2 of them, during the recording of the electrocardiogram, ST segment changes without pain occurred more frequently than the episodes of ST elevation with pain.

In one patient no significant difference, both in duration and in severity of the electrocardiographic abnormalities, was observed between the episodes with pain and those without pain, whereas in 3 patients a correlation between degree of electrocardiographic abnormality and pain was evident. Our electrocardiographic observations, therefore, do not provide definitive information to answer the question whether the changes occurring within the myocardium responsible for ST segment changes are identical or not to those eliciting pain.

From the haemodynamic study it was evident that during ST segment abnormalities the circulatory modifications were correlated to the degree of ST segment elevation.

Often patients with retrosternal chest pain occurring only at rest present a difficult diagnostic problem. In this form of angina, a detailed history, charting the time, degree, and circumstances of the attack is helpful in diagnosis. Even though a useful corroboration can be obtained with a single electrocardiographic recording taken promptly whenever pain occurs, the most detailed and direct information is provided by continuous electrocardiographic recording or monitoring. The importance (Robinson, 1965; Lunger and Shapiro, 1964) of these diagnostic means is further emphasized by these findings.

Myocardial infarction is described as likely to occur in the patients with Prinzmetal's variant angina. In the cases reported by Prinzmetal et al. (1959) and Robinson (1965) when the myocardial infarction occurred the attacks of anginal pain were immediately and

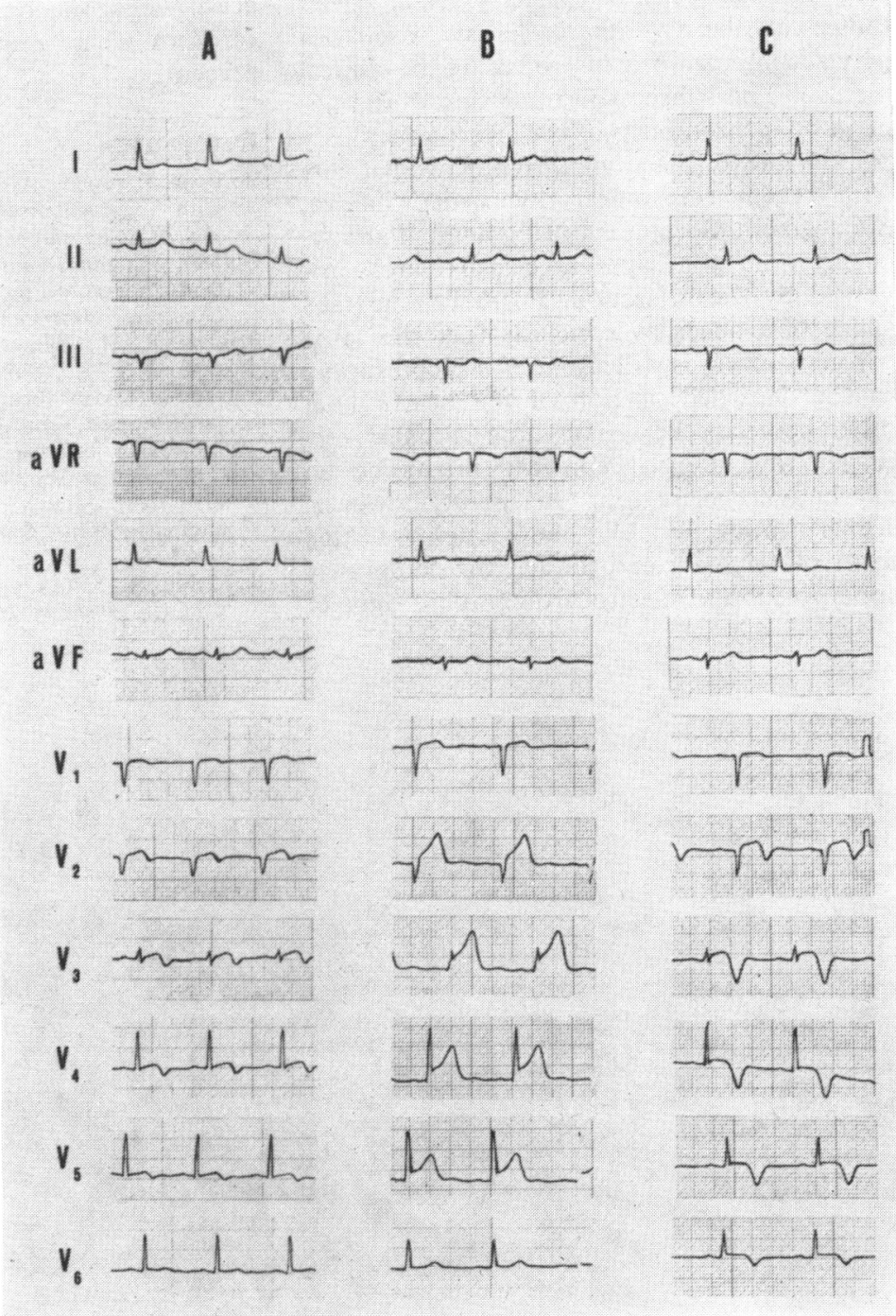

FI G. 4 (Case 4) Twelve-lead electrocardiogram of a patient with antero-septal myocardial infarction. $(A)$ in absence of anginal pain; $(B)$ at height of pain; $(C) 50$ seconds after the pain had subsided.

completely abolished. The two patients reported as Cases 3 and 4 a few months after the beginning of the anginal episodes developed myocardial infarction. In these patients, as in the case reported by Botti (I966), attacks of chest pain did not cease after myocardial infarction, but became more frequent and severe.

Temporary increased tonus of a large atherosclerotic coronary artery with a narrow lumen was suggested by Prinzmetal et al. (1959) as the cause of attacks of pain in this 
form of angina. Other coronary vessels, even though involved by atherosclerosis, would not play a significant role in producing the clinical picture. The subsequent infarction would depend on a thrombosis of the artery previously responsible for the angina. In our two patients with recent transmural myocardial infarct, ST segment elevations during anginal attacks occurred in the same myocardial areas involved by the infarction. From Prinzmetal's suggestion one may conclude that the same coronary artery is still a source of blood supply to the damaged portion of the heart. That other coronary vessels assume a major role in eliciting anginal episodes might be an alternative explanation. One point, however, seems unquestionable: an attack of this form of angina can produce the temporary superposition of electrocardiographic signs of acute ischaemia on the same areas of myocardium where signs of recent necrosis are present.

\section{References}

Botti, R. E. (1966). A variant form of angina pectoris with recurrent transient complete heart block. American fournal of Cardiology, 17, 443.

Lunger, M., and Shapiro, A. (I964). Continuous electrocardiographic monitoring in nocturnal angina. (Abstract.) American fournal of Cardiology, 13, I19.

Prinzmetal, M., Ekmekci, A., Kennamer, R., Kwoczynski, J. K., Shubin, H., and Toyoshima, H. (1960). Variant form of angina pectoris. Previously undelineated syndrome. Fournal of the American Medical Association, 174, 1794.

, Kennamer, R., Merliss, R., Wada, T., and Bor, N. (1959). Angina pectoris. I. A variant form of angina pectoris. American fournal of Medicine, 27, 375 .

Robinson, J. S. (1965). Prinzmetal's variant angina pectoris. American Heart fournal, 70, 797. 